\title{
Structural model explains high potency homoeopathic medicines and leads to a generalized understanding of medicines
}

\begin{abstract}
Conventional chemistry has no answer to either the secret of medicinal value of high potency (above 12c) homoeopathic medicines or how they can be identified, that is, differentiated from one another. Original medicinal substance being diluted away these are chemical-free medicines. This gives leverage to skeptics to brand cures by such medicines as placebo effect or mental cure. This article shows major contradictions in their hypothesis. Thereafter, as a logical derivation it arrives at the structural model: A substance is to be recognized as a medicine if it has the capability of curing disease(s) while its medicinal property is to be attributed to molecular structure of vehicle like water or of distinct chemical substance when it exists. It is a shift of focus from chemical formula to physical structure. Several works are cited to show that the no-chemical homoeopathic medicines have water structures. Structure, be it of crude medicinal substance or of molecular structure of water vehicle, plays the predominant role throughout the potency range. So, structures give us the generalized understanding of medicines. The article also shows that there is a link between structure, medicinal value and biological effect.
\end{abstract}

Keywords: Structural model; Chemical-free homoeopathic medicines; Generalized understanding of medicine; Homoeopathic medicines; Medicine's structure
Volume I Issue 2 - 2015

Chitta Ranjan Mahata

Indian Institute of Engineering Science and Technology, India

Correspondence: Chitta Ranjan Mahata, Hony Emesritus Scientist, IIEST, Flat-C2, 50/I College Road, Howrah-7I I 103,WB, Shibpur, India, Email crmahata@gmail.com

Received: April 17,20I5 | Published: June 23,20I5

\section{Introduction}

For absence of quantifiable original medicinal substance cures by high potency (above 12c), homoeopathic medicines are branded by skeptics as placebo effect or mental cure. It is a selfcontradictory hypothesis. For, mind also, to which they attribute cure, is not known to have any quantifiable active ingredient. It is also a frivolous hypothesis without description of the mechanism of action of mind for a cure. The skeptics mix up cure by chemical-free homoeopathic medicines with cure by transcendental powers of yogis and saints, which comes unknowingly even to ordinary individuals in some rare moments. But, in normal life people have to depend on medicines and not on transcendental powers coming from outside or sudden rise of that power from within for their cures. But, what is a medicine? A substance is to be recognized as a medicine if it has the capability of curing disease(s), chemical aspect not standing on its way. The chemical-free preparations of homoeopathy are also real medicines. The skeptics' denial in this respect originates from improper understanding of what is a medicine. A fully blown up placebo hypothesis means that medicines are non-essential for cures categorizing all cures as mental effects and all medicines as placebos. For, homoeopathy cannot be singled out against the spirit of impartiality of science and against accepting facts as facts. It is an inconvenient fact that chemical-free medicines of homoeopathy are benefiting us for more than two centuries. But open minded scientists do not deny facts. Contrary to the skeptics they try to find genuine explanations of facts. Further, the therapeutic benefit of homoeopathic medicines for babies, animals and plants proves the hollowness of the skeptics' view point. A new and unbiased approach is necessary to find how the chemical-free medicines of homoeopathy serve as real medicines. Following is such an attempt.

\section{Structural concept}

The chemical-free preparations of homoeopathy and nonhomoeopathic substances with identifying chemical formula BOTH cure diseases. We find that medicines may or may not have any distinct chemical formula. This forces us to review our concept of medicine. Logically, a substance is to be recognized as a medicine if it has the capability of curing disease. So, we must shift our focus from chemical formula and search for a new and generalized concept. This should not invalidate the prevalent concept of distinct chemical formula for medicines but add something new to accommodate the chemical-free substances as medicines. Without resorting to any wild guess let us look at some familiar facts. Amorphous carbon, Graphites and Diamond all have the same chemical formula. The difference lies in the structural arrangement of their constituent atoms. It leads to difference in their properties and difference in utility values. Then, in homoeopathy we have low potency Carbo Animalis and Graphites, where original medicinal substances are not diluted away. These are two different medicines with same chemistry. How can we explain it? It is only by difference in structures. Structural change leads to change of medicine even with same chemistry. Structure gets an upper hand in deciding the medicinal value. Medicinal property is to be attributed to molecular structure of the 'substance'. In lower potencies (below 12c) the 'substance' may be the original medicinal substance. But, in higher potencies (above 12c) there is no other 'substance' except the vehicle. Differences in medicinal values of the above two medicines is maintained in higher potencies. Side by side they have differences in the molecular structures of the vehicle like water as shown in the next section by which they can be identified or differentiated from one another. Further, in a medicine like X-ray medicinal value in all potencies can come only from structure of vehicle molecules. Everywhere we find that 'structures' serve as the real medicine. As a logical derivation this gives us the structural model of medicine: A substance is to be recognized as a medicine if it has the capability of curing disease while its medicinal property is to be attributed to molecular structure of vehicle like water or of distinct chemical substance when it exists. Note that we have shifted our emphasis from chemical formula to physical structure. 
For strengthening this proposition (namely, structural concept) let us examine how water can have the potential for such a role. Is it possible for water as a vehicle to have stable structures which can serve as medicines? Can water have enough structural variations corresponding to the vast number of homoeopathic medicines along with their potencies? Can water structures be influenced by solvated molecules in it? Here are the supporting facts:

a. At room temperature ordinary water contains innumerable tiny floating ice crystals or icicles. ${ }^{1-3}$ This satisfies the requirement of stable structure.

b. Forms of these crystals are so varied in number that practically no two ice crystals are identical. ${ }^{2}$ This satisfies the requirement of vast number of forms of structures.

The shape and size of these molecular clusters are influenced by impurities, ions of other substances and even large foreign molecules. ${ }^{2,4}$ This satisfies the requirement of induced structures in various potencies. Thus we find that water has the requisite potential also. The three special properties of water enlisted above serve as the basis of the structural concept. Many investigations have been done on water, a good listing being provided by Martin Chaplin. ${ }^{5}$ with more than 2,050 references including a homoeopathy-related review. ${ }^{6}$ But the properties mentioned above continue to remain un-contradicted.

\section{Supports for structural concept}

Possibly Barnard GO. ${ }^{7}$ was the first to hint at the structural concept though he named it as water polymer. But, his work lacked experimental support. Stupendously successful experimental results were given by WE Boyd. ${ }^{8}$. But, explanation for the emanations he detected by his 'emanometer' were missing at that time. Much later Chattopadhyay $\mathrm{R}$ et al. ${ }^{9}$ offered an explanation suggesting that it originated from structured vehicle getting excitation from ambient electromagnetic radiation. Anagnostatos GS et al. ${ }^{10}$ also proposed his structural model calling the structured water (of vehicle of potentisation) as 'clathrates'. He described how clathrates are likely to be generated at different stages of serial dilution. Maity T et al. ${ }^{11,12}$ and Mahata $\mathrm{CR}$ et al. ${ }^{13,14}$ show experimentally that water structures are present in medicines both below and above the Avogadro limit of 12c Rustum R et al. ${ }^{15,16}$ firmly supports the structural concept with some experimental results. Benveniste A et al. ${ }^{17}$ in their experiments with human basophile degranulation show that 'memory' of the original substance are retained in serial dilution followed by vortexing even beyond the Avogadro limit. It is not a work with medicines but it has relevance to the dilution/potentisation of homoeopathy. They call it as 'water memory', but we like to call it induced structure. Similarly, Louis $\mathrm{R}^{18}$ in his investigation with dilution of substance like $\mathrm{LiCl}$ and $\mathrm{NaCl}$ shows that the hydrogen-bonded network of water remains modified even in dilutions beyond Avogadro limit. We may assume that such modification will lead to variation in structure of molecular clusters of the vehicle. Montagnier L et al. ${ }^{19}$ demonstrated that even massively diluted (10 raised to the power 12) DNA and RNA solutions continue to emit electromagnetic radiations like the un-diluted one. Its possible explanation is offered in ref $^{9}$ in terms of structures. Latest support to structural model comes from the work of Elia $\mathrm{V}$ et al. ${ }^{20}$ The structural model may be at its infancy due to limited data in its support. But, we may consider it to be a beginning with a promise.

\section{Link between structures and medicinal value and biological effect}

Due to various reasons the molecules of a living organism may change their form to some extent. But when this develops to a considerable extent it may be considered to be in a diseased state. These damaged molecules can be repaired with the help of tiny ice crystals. By freezing onto curved molecules the icicles straighten them out; giving them their usual configuration. ${ }^{3}$ The action of water structures on bio-molecules is supported by the following facts:

a. All healthy bio-molecules fit in nicely within the hollows of water molecules in solid as well as liquid forms. Otherwise we could not preserve foods in refrigerators. But the reaction of water towards the molecules whose form does not fit the structure of ice is quite different - it breaks the larger molecules and drives away the small ones. For this reason, the ice in Arctic Ocean is fresh-water ice -- free from all salts. ${ }^{3}$ So, it may safely be assumed that water structures of potentised medicines will suitably bend bio-molecules to get a desired fit.

b. Structural fitting and template principle explain biological metabolic processes. This is quite well known to biologists. So, we are not assuming anything new. We are only saying that water structures of medicines can also serve as templates.

c. Water of diseased human cells is found to be rather disordered as compared to water of healthy cells. . $^{3,21-25}$ Introduction of suitable water structure into the body is, therefore, very likely to bring the diseased bio-molecules to their normal condition and thereby restore health.

It should be carefully noted here that any arbitrary water structure will not be a medicine. A radio set may be placed in a location full of all sorts of electromagnetic waves. But, the set will respond only to the frequency of the electromagnetic signal to which it is tuned. Similarly, structural influence (and hence, medical action) can occur only with closeness of structural matching with bio-molecules. Otherwise, it becomes a misfire. In fact, this is very likely to be root cause for difficulty in selecting homoeopathic medicines.

That structures like bio-templates play important role in metabolic process is an established fact in biology. DNAs and RNAs are recognized as primary and secondary templates. But, when it comes to medicines either homoeopathic or non-homoeopathic nobody thought of considering structures. People are prone to think of medicines in terms of their chemical composition. But, every chemical formula has its corresponding physical structure. We think of chemical reaction in terms of chemical formula. Why can't we consider chemical reaction as reaction between structures? The present author thinks that it is a better approach. It does not invalidate conventional thinking but enable us to interpret all medicinal actions successfully.

\section{Conclusion}

The title of the article is the conclusion - Structural model explains high potency homoeopathic medicines and leads to a generalized understanding of medicines.

\section{Acknowledgments}

None. 


\section{Conflict of interest}

The authors declare that there is no conflict of interest.

\section{Funding}

None.

\section{References}

1. Karapetyants M, Drakin S. Structure of Matter. Russia: Mir Publishers, Moscow; 1974. pp. 258-259; 285; 295-297.

2. Finkelnburg W. Structure of Matter. New York, USA: Academic Press; 1964. pp. 412-414.

3. Sergeev B. Physiology for Everyone. Moscow, Russia: Mir Publishers; 1973. pp. 11-16.

4. Singh PP, Chhabra HL. Topological investigation of the ethanol/water system and its implication for the mode of action of Homoeopathic Medicines. Homeopathy. 1993;82(3):164-171.

5. Chaplin M. Water Structure and Science Site Map. UK: Water structure and Science; 2015.

6. Chaplin M. Homeopathy. UK: Water structure and Science; 2015.

7. Barnard G. Microdose paradox - a new concept. J Am Homeopath. 1965;58(7):205-212.

8. Horders T. The Electronic Reactions Of Abrams: Communication To The Royal Society Of Medicine By A Committee Of Which Sir Thomas Horder Is Chairman. Br Med J. 1925;1(3343):179-185.

9. Chattopadhyay R, Sadhukhan M, Pal A, et al. On electromagnetic signals from vastly diluted DNA solutions, potentised medicines and even metals. Wesleyan Journal of Research . 2012;5(1):50-55.

10. Anagnostatos GS, Vithoulkas G, Garzonis P, et al. working hypothesis for homoeopathic microdiluted remedies. The Berlin Journal of Research in Homoeopathy. 1991;1(3):141-147.

11. Maity T, Ghosh D, Mahata CR. Theory and Instrumentation related to Potentised Homoeopathic Medicines. Indian Journal of Research in Homoeopathy. 2007;2(3):1-15.

12. Maity T, Ghosh D, Mahata CR. Effect of Dielectric Dispersion on Potentised Homoeopathic Medicines. Homeopathy. 2010;99(2):99-103.
13. Mahata CR. Dielectric Dispersion Studies Indicate Change in Structure of Water by Potentised Homeopathic Medicines. Journal of The Institution of Engineers. 2013;93(4):231-35.

14. Mahata CR. Dielectric Dispersion Studies of Some Potentised Homoeopathic Medicines Reveal Structured Vehicle. Homeopathy. 2012;102(4):262-267.

15. Roy R, Tiller W A, Iris B, et al. The structure of liquid water; novel insights from materials research; potential relevance to homeopathy. Indian Journal of Research in Homoeopathy. 2005;2(3):1-24.

16. Rao ML, Roy R, Bell IR, et al. The defining role of structure (including epitaxy) in the plausibility of homeopathy. Homeopathy. 2007;96(3):175-182.

17. Davenas E1, Beauvais F, Amara J, et al. Human basophil degranulation triggered by very dilute antiserum against IgE. Nature. 1988;333(6176):816-818.

18. Rey L (2003) Thermo-luminescence of ultra-high dilutions of lithium chloride and sodium chloride. Physica A: Statistical Mechanics and its Applications 323:67-74.

19. Montagnier L, Aïssa J, Ferris S, et al. Electromagnetic signals are produced by aqueous nanostructures derived from bacterial DNA sequences. Interdiscip Sci. 2009;1(2):81-90.

20. Elia V, Ausanio G, Gentile F, et al. Experimental evidence of stable water nanostructures in extremely dilute solutions, at standard pressure and temperature. Homeopathy. 2014;103(1):44-50.

21. Chaughule RS. NMR: A New Eye on Cancer. Science Today. 1978;2327.

22. Damadian R. Tumor Detection by NMR. Science. 1971;171:1151-1153.

23. Hollis DP, Economou JS, Parks LC, et al. Nuclear Magnetic Resonance Studies of several experimental and human malignant tumors. Cancer Res. 1973;33:2156-2160.

24. Chaughule RS. Normal and Malignant Tissues:An Investigation by Pulsed NMR. Indian J of Biochem and Biophy. 1974;11:256.

25. Ranade SS, Shah S, Korgaonkar KS, et al. Absence of Correlation between Spin-Lattice Relaxation Times and water content in human tumor tissues. Physiol Chem Phys. 1976;8(2):131-134. 